\title{
Recent studies that influence the chemotherapeutic paradigm in the management of advanced ovarian cancer
}

\author{
Maurie Markman
}

\begin{abstract}
Address: Department of Gynecologic Medical Oncology, The University of Texas MD Anderson Cancer Center, 1515 Holcombe Boulevard, Houston, TX 77030, USA

Email: mmarkman@mdanderson.org
\end{abstract}

FI000 Medicine Reports 2010, 2:29 (doi:10.3410/M2-29)

The electronic version of this article is the complete one and can be found at: http://fl000.com/reports/medicine/content/2/29

\begin{abstract}
Several recently reported evidence-based phase 3 trials that focus on the role of cytotoxic chemotherapy have the potential to strongly influence the care of women with advanced epithelial ovarian cancer. Particular areas of interest include: (a) neoadjuvant chemotherapy; (b) chemotherapy of recurrent platinum-sensitive ovarian cancer; and (c) the relevance of paclitaxel scheduling in the primary chemotherapeutic management of the malignancy. This report will briefly discuss the results of these studies and the potential implications of the findings for routine care of patients in these clinical settings.
\end{abstract}

\section{Introduction and context}

An attempt at optimal surgical cytoreduction prior to the initiation of cytotoxic chemotherapy has long been recognized as the standard-of-care management strategy in women presenting with advanced epithelial ovarian cancer $[1,2]$. However, multiple reports over the course of more than a decade have suggested that in carefully selected patients the administration of chemotherapy prior to an attempt at surgery (neoadjuvant chemotherapy) is an acceptable or perhaps even a superior option (reduced treatment-related morbidity) $[3,4]$.

Standard treatment of advanced ovarian cancer includes a platinum agent plus a taxane [1], with most oncologists employing a regimen of carboplatin plus either paclitaxel or docetaxel. Of note, the taxane in these programs has been routinely administered on an every-3-week schedule.

Several previously published phase 3 randomized trials have revealed the superiority of combination platinum-based chemotherapy in recurrent ovarian cancer (platinum-free interval $>6$ months) compared with single-agent platinum $[5,6]$. However, it remained unknown whether there would be any difference in outcome depending on which known biologically active drug in ovarian cancer is added to platinum (generally carboplatin) in a two-drug combination strategy (e.g., docetaxel, gemcitabine, paclitaxel, pegylated liposomal doxorubicin [PLD], and topotecan).

\section{Recent advances}

In what is likely to be considered a landmark study in the gynecologic cancer arena, a multinational cooperative group effort directly compared (phase 3 randomized trial) primary surgery followed by chemotherapy ('control arm') with an experimental neoadjuvant approach (chemotherapy followed by interval surgical cytoreduction) in women with large-volume intra-abdominal epithelial ovarian cancer [7]. The study revealed no difference in overall survival between the two study arms, with less serious therapy-associated morbidity (and mortality) for women randomly assigned to the neoadjuvant approach.

The trial has received criticism due to the overall poor survival of the study population and questions of the adequacy of surgery (low rate of optimal debulking) across the multiple participant institutions and 
countries. Particular strengths of the study include the excellent design (phase 3 randomized trial) and conduct, stratification by country (an important response to the issue of the adequacy of surgery), and the observation that there was no suggestion of a survival benefit associated with the primary surgical approach.

The results of the recently reported CALYPSO (Caelyx [pegylated liposomal doxorubicin hydrochloride] in Platinum-Sensitive Ovarian Patients) trial have provided provocative evidence supporting the superiority of the combination of carboplatin plus PLD compared with carboplatin plus paclitaxel in this clinical setting [8]. The study, designed as a non-inferiority phase 3 randomized trial, demonstrated a statistically significant improvement in median progression-free survival (11.3 versus 9.4 months; $P=0.005$; hazard ratio [HR] 0.821) in favor of the PLD-containing regimen. Data regarding overall survival were not mature at the time of the initial presentation of the efficacy results. Perhaps even more provocative than the interesting survival data was the observation that patients treated with the carboplatin/ PLD program experienced an impressively lower risk for the development of carboplatin-associated hypersensitivity reactions and a corresponding reduced risk of having treatment on the protocol discontinued due to excessive toxicity [9].

As solid (although certainly not definitive) data support the conclusion that the platinum agents are the single most biologically active drugs in ovarian cancer (including in the platinum-sensitive second-line setting), it is possible that the observed superiority of the carboplatin/PLD regimen (compared with carboplatin/paclitaxel) resulted, at least in part, from the ability of a larger percentage of patients with potentially platinum-sensitive tumors to receive an optimal number of cycles of carboplatin.

While this concept must be considered only hypothesisgenerating, the available data certainly support the conclusion. Furthermore, a previously reported small phase 3 randomized trial conducted by the Southwest Oncology Group that compared single-agent carboplatin with the combination of carboplatin plus PLD revealed a similar striking reduction in the incidence of carboplatinassociated hypersensitivity reactions in the two-drug regimen $[10,11]$.

An acceptable biological explanation for this observation remains to be established. However, it is conceivable that the pegylated liposomal preparation itself may have some impact on the development of clinically relevant hypersensitivity. This important observation requires further investigation as the negative impact associated with the sudden onset of serious carboplatin allergic reactions is well known [9].

A recently reported randomized phase 3 trial conducted by the Japanese Gynecologic Oncology Group revealed the superiority of carboplatin plus weekly paclitaxel combined with every-3-week carboplatin compared with the same dose and schedule of the platinum agent but with paclitaxel administered on the 'standard' every-3-week program [12]. The weekly paclitaxel strategy was associated with improved progression-free survival (median 28 versus 17.2 months; $P=0.0015$; HR 0.71 ) and 3 -year overall survival $(72.1 \%$ versus $65.1 \% ; P=0.03$; HR 0.75$)$.

\section{Implications for clinical practice}

The data obtained in the landmark phase 3 neoadjuvant chemotherapy trial support the conclusion that, in carefully selected patients with advanced ovarian cancer (e.g., extensive disease requiring bowel resection to achieve an optimal residual state or the presence of comorbidity increasing the risks associated with aggressive cytoreductive surgery), primary chemotherapy followed by an attempt at surgical cytoreduction (when appropriate) is an acceptable management strategy. Future research in this area, including exploration of a possible role for intraperitoneal chemotherapy following successful interval surgical cytoreduction, is clearly indicated. Of note, a phase 3 multinational cooperative group trial examining this specific issue has recently been activated.

The results of the CALPYSO trial reveal that the combination of carboplatin plus PLD is an acceptable treatment strategy in potentially platinum-sensitive recurrent ovarian cancer.

While it will be important for the potentially paradigmchanging data in the Japanese front-line weekly paclitaxel trial to be confirmed in a second phase 3 study, highly relevant support for the superiority of weekly paclitaxel comes from two recently reported randomized breast cancer trials (metastatic disease and adjuvant treatment) that revealed the superiority of weekly paclitaxel administration compared with the 'standard' every-3-week regimen $[13,14]$. Considered together, the available data strongly support the conclusion that carboplatin plus weekly paclitaxel is an acceptable and likely superior regimen for the chemotherapeutic management of advanced ovarian cancer.

\section{Abbreviations}

HR, hazard ratio; PLD, pegylated liposomal doxorubicin.

\section{Competing interests}

The author declares that he has no competing interests. 


\section{References}

I. Hennessy BT, Coleman RL, Markman M: Ovarian cancer. Lancet 2009, 374: | 37|-82

2. Bristow RE, Tomacruz RS, Armstrong DK, Trimble EL, Montz FJ: Survival effect of maximal cytoreductive surgery for advanced ovarian carcinoma during the platinum era: a meta-analysis. J Clin Oncol 2002, 20:1248-59.

3. Schwartz PE, Rutherford TJ, Chambers JT, Kohorn El, Thiel RP: Neoadjuvant chemotherapy for advanced ovarian cancer: long-term survival. Gynecol Oncol 1999, 72:93-9.

4. Vergote I, De Wever I, Tjalma W, Van Gramberen M, Decloedt J, van Dam P: Neoadjuvant chemotherapy or primary debulking surgery in advanced ovarian carcinoma: a retrospective analysis of 285 patients. Gynecol Oncol 1998, 71:431-6.

5. Pfisterer J, Plante M, Vergote I, du Bois A, Hirte H, Lacave AJ, Wagner U, Stähle A, Stuart G, Kimmig R, Olbricht S, Le T, Emerich J, Kuhn W, Bentley J, Jackisch C, Lück HJ, Rochon J, Zimmermann AH, Eisenhauer E; AGO-OVAR; NCIC CTG; EORTC GCG: Gemcitabine plus carboplatin compared with carboplatin in patients with platinum-sensitive recurrent ovarian cancer: an intergroup trial of the AGO-OVAR, the NCIC CTG, and the EORTC GCG. J Clin Oncol 2006, 24:4699-707.

6. Parmar MK, Ledermann JA, Colombo N, du Bois A, Delaloye JF, Kristensen GB, Wheeler S, Swart AM, Qian W, Torri V, Floriani I, Jayson G, Lamont A, Tropé C; ICON and AGO Collaborators: Paclitaxel plus platinum-based chemotherapy versus conventional platinum-based chemotherapy in women with relapsed ovarian cancer: the ICON4/AGO-OVAR-2.2 trial. Lancet 2003, 36 I:2099-106.

7. Vergote I, Tropé CG, Amant F, Kristensen GB, Sardi JE, Ehlen T, Johnson N, Verheijen RH, Van der Burg ME, Lacave AJ, Benedetti Panici P: EORTC-GCG/NCIC-CTG randomised trial comparing primary debulking surgery with neoadjuvant chemotherapy in stage IIIC-IV ovarian, fallopian tube and peritoneal cancer (OVCA). Paper presented at IGCS I2th Biennial Meeting: 25-28 October 2008; Bangkok, Thailand. Abstract 1767.

8. Pujade-Lauraine E, Mahner S, Kaern J, Gebski V, Heywood M, Vasey P, Reinthaller A, Vergote I, Pignata S, Ferrero A: A randomized, phase III study of carboplatin and pegylated liposomal doxorubicin versus carboplatin and paclitaxel in relapsed platinumsensitive ovarian cancer (OC): CALYPSO study of the Gynecologic Cancer Intergroup (GCIG) [abstract]. J Clin Oncol 2009, 27(Suppl): I5s. Abstract LBA5509.
9. Zanotti KM, Markman M: Prevention and management of antineoplastic-induced hypersensitivity reactions. Drug Saf 200I, 24:767-79.

10. Alberts DS, Liu PY, Wilczynski SP, Clouser MC, Lopez AM, Michelin DP, Lanzotti V], Markman M; Southwest Oncology Group: Randomized trial of pegylated liposomal doxorubicin (PLD) plus carboplatin versus carboplatin in platinum-sensitive (PS) patients with recurrent epithelial ovarian or peritoneal carcinoma after failure of initial platinum-based chemotherapy (Southwest Oncology Group Protocol S0200). Gynecol Oncol 2008, 108:90-4.

II. Markman M, Moon J, Wilczynski S, Lopez AM, Rowland KM Jr, Michelin DP, Lanzotti VJ, Anderson GL, Alberts DS: Single agent carboplatin versus carboplatin plus pegylated liposomal doxorubicin in recurrent ovarian cancer: final survival results of a SWOG (S0200) phase 3 randomized trial. Gynecol Oncol 2010, I 16:323-5.

12. Katsumata N, Yasuda M, Takahashi F, Isonishi S, Jobo T, Aoki D, Tsuda H, Sugiyama T, Kodama S, Kimura E, Ochiai K, Noda K; Japanese Gynecologic Oncology Group: Dose-dense paclitaxel once a week in combination with carboplatin every 3 weeks for advanced ovarian cancer: a phase 3, open-label, randomised controlled trial. Lancet 2009, 374:|33|-8.

FI000 Factor 8.6 Exceptional

Evaluated by Maurie Markman 06 Oct 2009, Jim Orr 09 Oct 2009, Jonathan Ledermann 22 Oct 2009, Rob Burger 17 Dec 2009, Paola Gehrig 02 Mar 2010

13. Seidman AD, Berry D, Cirrincione C, Harris L, Muss H, Marcom PK, Gipson G, Burstein H, Lake D, Shapiro CL, Ungaro P, Norton L, Winer E, Hudis C: Randomized phase III trial of weekly compared with every-3-weeks paclitaxel for metastatic breast cancer, with trastuzumab for all HER-2 overexpressors and random assignment to trastuzumab or not in HER-2 nonoverexpressors: final results of Cancer and Leukemia Group B protocol 9840. J Clin Oncol 2008, 26:1642-9.

Changes Clinical Practice

FI000 Factor 6.0 Must Read

Evaluated by Gilberto Schwartsmann 29 Apr 2008

14. Sparano JA, Wang M, Martino S, Jones V, Perez EA, Saphner T, Wolff AC, Sledge GW Jr, Wood WC, Davidson NE: Weekly paclitaxel in the adjuvant treatment of breast cancer. $N$ Engl J Med 2008, 358:|663-7|.

FI000 Factor 6.4 Must Read

Debu Tripathy 02 May 2008, Generosa Grana 29 Aug 2008 